\title{
Appell an die medizinischen Fachgesellschaften
}

\section{Christian Rathgeb}

Dr. iur., Regierungsrat Kanton Graubünden, Vorsteher des Departements für Justiz, Sicherheit und Gesundheit

\section{Entscheide der medizinischen Fach- gesellschaften wirken sich auf die Gesundheitsversorgung der Kantone aus}

Im Gesundheitswesen werden auf nationaler Ebene zunehmend Entscheide getroffen oder Richtlinien erlassen, die für periphere Kantone mit geringer Bevölkerungszahl und einer dezentralen Besiedelung wie den Kanton Graubünden einschneidende Auswirkungen auf die Gesundheitsversorgung der Bevölkerung wie auch der Feriengäste zeitigen.

Entsprechende Entscheide oder Richtlinien werden dabei nicht nur vom Bund getroffen beziehungsweise erlassen. Auch Entscheide beziehungsweise Richtlinien der medizinischen Fachgesellschaften können gravierende Auswirkungen auf die Gesundheitsversorgung der Kantone haben. Zumindest bei einzelnen Entscheiden und Richtlinien besteht Grund zur Annahme, dass sie nicht qualitativ, sondern standespolitisch oder in der zentrumsspitallastigen Zusammensetzung des Entscheidgremiums begründet sind. So ist beispielsweise nicht nachvollziehbar, welchen Beitrag die minimalen Netto- und Brutto-Flächenvorgaben pro Bett einer Station, wie sie in den von den Vorständen verschiedener Fachärztegesellschaften im Mai 2013 verabschiedeten Richtlinien für die Anerkennung von Intermediate Care Units enthalten sind, zur Qualität leisten sollen. Der Kanton Graubünden sah sich auf Grund der räumlichen Verhältnisse in den Spitälern jedenfalls gezwungen, die minimalen Netto- und Bruttoflächenvorgaben pro Bett der Intermediate Care Units zu reduzieren.

Die zentrumsspitallastige Zusammensetzung der fachärztlichen Gremien ist oft darin begründet, dass es den in kleineren Spitälern tätigen Ärztinnen und Ärzten oft mangels Stellvertretung nicht möglich ist, an den Fachärztetagungen teilzunehmen, womit die Interessen beziehungsweise Anliegen der kleineren Spitäler oft ungehört bleiben. Es ist entsprechend seitens der medizinischen Fachgesellschaften zu überlegen, wie die Ärzte der kleineren Spitäler besser in ihre Entscheidfindung eingebunden werden können.

\section{Bedürfnisse der Kantone mit einer dezentralen Besiedelung sind bei der Konzeption der Weiterbildungs- programme miteinzubeziehen}

Durch die zunehmende Spezialisierung in der Medizin gestaltet sich die Rekrutierung von Assistenzärzten und Fachärzten für peripher gelegene kleine Regionalspitäler immer schwieriger. Eine unzureichende ärztliche Dotierung beeinträchtigt oder gefährdet gar die medizinische Grundversorgung im Einzugsgebiet dieser Spitäler.

Bei der Konzeption der Weiterbildungsprogramme insbesondere in den Gebieten Allgemeine Innere Medizin und Allgemeinchirurgie sollten die Fachärztegesellschaften entsprechend dem Umstand Rechnung tragen, dass weitläufige Kantone mit einer dezentralen Besiedelung neben spezialisierten Ärzten im Zentrumsspital für die medizinische Grundversorgung ihrer Bevölkerung über das ganze Kantonsgebiet in den übrigen Spitälern Ärzte mit einer breiten Ausbildung benötigen. Es gibt immer wieder Notfälle, wo der $\mathrm{Pa}$ tient nicht verlegbar ist oder bei denen der Patient bei einer Verlegung aus dem therapeutischen Zeitfenster fällt (z.B. bei der Schlaganfalltherapie). Damit Ärzte in den Regionalspitälern bei Notfällen über die erforderliche Erfahrung verfügen, müssen sie über eine breite Grundausbildung verfügen und diese im Tagesgeschäft auch zur Anwendung bringen dürfen.

\section{Appell an die medizinischen Fachgesellschaften}

In meiner Eigenschaft als Gesundheitsdirektor eines Kantons mit einer dezentralen Gesundheitsversorgung appelliere ich an die Fachärztegesellschaften zu bedenken, welche Auswirkungen ihre Entscheide beziehungsweise Richtlinien auf die Versorgung eines dünn besiedelten Gebirgskantons haben können, und entsprechend ihre Entscheide und Richtlinien so auszugestalten, dass sie auch den Gegebenheiten und Bedürfnissen eines solchen Kantons Rechnung tragen. 